\title{
Evaluation of clinical pharmacist interventions for adverse events in hospitalized patients with thoracic cancer receiving cancer chemotherapy
}

\author{
HIROTOSHI IIHARA ${ }^{1,2}$, CHIEMI HIROSE ${ }^{1}$, NORIHIKO FUNAGUCHI ${ }^{3,4}$, JUNKI ENDO $^{3}$, \\ FUMITAKA ITO $^{3}$, KOMEI YANASE $^{3}$, DAIZO KAITO $^{3}$, YASUSHI OHNO $^{3}$ and AKIO SUZUKI ${ }^{1,2}$ \\ ${ }^{1}$ Department of Pharmacy, Gifu University Hospital, Gifu 501-1194; ${ }^{2}$ Laboratory of Pharmacy Practice and \\ Social Science, Gifu Pharmaceutical University, Gifu 501-1196; ${ }^{3}$ Department of Cardiology and Respiratory \\ Medicine, Gifu University Graduate School of Medicine, Gifu 501-1194; ${ }^{4}$ Department of Respiratory Medicine, \\ Asahi University Hospital, Gifu 500-8856, Japan
}

Received August 12, 2019; Accepted February 12, 2021

DOI: $10.3892 / \mathrm{mco} .2021 .2278$

\begin{abstract}
Due to the increasing complexity of cancer chemotherapy and its associated supportive care, the role of clinical pharmacists in cancer chemotherapy is becoming increasingly more important. The present study evaluated the clinical interventions of a single pharmacist on the adverse events in hospitalized patients with thoracic cancer receiving cancer chemotherapy. A single-center, retrospective study was conducted at the 614-bed, tertiary care Gifu University Hospital. Hospitalized patients with thoracic cancer who received cancer chemotherapy in the respiratory medicine ward between April 2013 and May 2014 were enrolled. One of the two clinical pharmacists in charge was based in the respiratory medicine ward and implemented pharmaceutical care for the patients, including management of adverse events. Patient data were recorded in the electronic medical chart and retrospectively analyzed. A total of 445 patients with thoracic cancer received cancer chemotherapy in the respiratory medicine ward. A total of 152 interventions (101 patients) were performed by the clinical pharmacist prior to the administration of cancer chemotherapy, half of which comprised the addition of drugs to prevent adverse events. A total of 190 patients (39.4\%) experienced grade $\geq 2$ non-hematological or grade $\geq 3$ hematological adverse events associated with cancer chemotherapy, and 223 medical interventions for relief of adverse events lowered the incidence of grade $\geq 2$ non-hematological or grade $\geq 3$ hematological adverse events to $17.8 \%$. Of these, 45.3 and $7.5 \%$ of medical interventions for non-hematological and hematological adverse events,
\end{abstract}

Correspondence to: Dr Akio Suzuki, Department of Pharmacy, Gifu University Hospital, 1-1 Yanagido, Gifu 501-1194, Japan

E-mail: akio@gifu-u.ac.jp

Key words: adverse events, hospitalization, clinical pharmacists, cancer chemotherapy, medical intervention respectively, were implemented based on the pharmacist's recommendations. These findings revealed the marked contribution of a single clinical pharmacist in the respiratory medicine ward to the prevention and relief of adverse events in hospitalized patients with thoracic cancer receiving cancer chemotherapy.

\section{Introduction}

Adverse events are a major medical problem in hospitalized patients $(1,2)$. In particular, adverse events are frequently observed in patients receiving cancer chemotherapy because of the narrow therapeutic range of anticancer drugs, which are associated with a high incidence of toxicity $(3,4)$. Adverse events often become severe, resulting in dose delays and reductions, therapy discontinuation, or decreased quality of life, all of which negatively influence the therapeutic effect (5-7). Therefore, it is necessary to consider dose adjustment or withdrawal of anticancer drugs depending on bone marrow function, renal function, liver function and the state of occurrence of adverse events in previous treatment in many cancer chemotherapy regimens (8). Additionally, the prevention and timely remedy of adverse events in patients is of the importance. However, many chemotherapy regimens are highly complex, including prophylactic supportive care, and require frequent dosage adjustments, which can lead to prescription errors $(9,10)$. Therefore, medical care related to the administration of cancer chemotherapy should be performed by a team of medical staff comprising physicians, nurses, clinical pharmacists, nutritionists and other medical staff $(11,12)$.

Clinical pharmacists play a critical role in pharmaceutical practices in cancer chemotherapy, including reviewing cancer chemotherapy regimens, dose adjustments for organ dysfunction, weight, age, monitoring for drug-drug interactions, verifying prescription orders containing anticancer drugs, monitoring efficacy and adverse events, preventing or alleviating adverse events, implementing palliative care and providing drug information to medical staff $(13,14)$. Even 
though clinical pharmacists spend a considerable amount of time ensuring patient safety during the preparation and dispensing of chemotherapy, only a few studies have evaluated their efforts related to management in patients receiving cancer chemotherapy $(15,16)$.

The purpose of this retrospective study was to evaluate a single pharmacist's interventions on the rate of adverse events in hospitalized patients with thoracic cancer receiving cancer chemotherapy.

\section{Materials and methods}

Study design and setting. We conducted a single-center, retrospective study at the 614-bed, tertiary care Gifu University Hospital. We enrolled hospitalized patients with thoracic cancer who received cancer chemotherapy in the respiratory medicine ward between April 2013 and May 2014. One of two clinical pharmacists in charge, including one oncology pharmacy specialist, was based in the respiratory medicine ward and implemented pharmaceutical care for the patients. Adverse events were monitored in cooperation with physicians, nurses and clinical pharmacists and were recorded in each patient's electronic medical chart. Appropriate drug management for adverse events was performed by the physician based on his/her own judgement or on the pharmacist's recommendation, and judgment of whether or not the intervention improved an adverse event was continued until the end of the treatment.

Ethics statement. The present study was conducted according to the guidelines for human studies of the ethics committee of Gifu University Graduate School of Medicine and the Government of Japan, and was approved by the university's institutional review board (approval no. 28-348). In view of the retrospective nature of the study, informed consent from the subjects was not mandated.

Cancer chemotherapy. In our hospital, all thoracic cancer chemotherapy is administered based on a treatment protocol agreed upon by physicians and clinical pharmacists in advance. This protocol sets the criteria for starting treatment, dose reduction or drug withdrawal accompanying incidence of adverse events and dose adjustments for renal and hepatic function based mainly on evidence from original reports, and prophylactic supportive care, including antiemetic medication; hydration management for cisplatin; folic acid supplements and vitamin $\mathrm{B}_{12}$ injections for pemetrexed; glucocorticoid and histamine $\mathrm{H}_{1}$ for paclitaxel hypersensitivity; and hydration while on having renal dysfunction. Standard antiemetic medication was administered based on the Japanese Society of Clinical Oncology guidelines (17). Additionally, patients who developed chemotherapy-induced nausea and vomiting were prophylactically administered aprepitant, olanzapine, lorazepam or prochlorperazine in addition to standard prophylactic antiemetic therapy at the next scheduled treatment.

Standardized chemotherapy order forms containing the dosage of anticancer drug and dose calculation $\left(\mathrm{mg} / \mathrm{m}^{2}\right)$, start date and time, day of therapy, solution diluent and volume, infusion rate (drips), route (intravenous push or infusion), duration of infusion, frequency of anticancer drug administration, total number of scheduled doses, and prophylactic supportive care restricted to injections were entered into the electronic medical chart for each regimen.

Duties of clinical pharmacists. Clinical pharmacists performed the following duties: Interviewed all patients on admission and documented medications brought to the hospital and patients' medication history, daily review of laboratory data, verified prescriptions, monitored and managed adverse events, provided drug information to medical staff, and patient education.

Clinical pharmacists provided physicians with timely information and advice on adverse events; drug interactions; and appropriate dosages, dose intervals, and routes of administration. All interventions performed by pharmacists were recorded, including whether or not their recommendations were accepted by the physicians.

Assessment and intervention of adverse events. In the present study, adverse events were defined as harm due to medications (adverse drug event), radiation therapy, or events that occurred during the course of the disease, excluding those due to medical errors, system errors and equipment failure. The severity of adverse events was graded according to the Common Terminology Criteria for Adverse Events (National Cancer Institute, MD, USA) version 4.0. Pharmacotherapy for adverse events was based on the National Comprehensive Cancer Network guidelines for supportive care and other clinical practice guidelines for each adverse event.

Interventions for adverse events were carried out in patients showing grade $\geq 2$ non-hematological or grade $\geq 3$ hematological adverse events, and the effect of intervention was evaluated before and after intervention. The judgment whether or not the intervention improved the adverse events was conducted until the end of the treatment.

Data analysis. The following patient data were recorded in specially prepared Microsoft Excel 2010 (Microsoft Corp.) spreadsheets: Patient age and sex; date of admission and discharge; diagnosis; purpose of hospitalization; list of private medications; clinical pharmacists' prescription proposals; and adverse events, their grade, and outcome of intervention. The duration of hospital stay was documented in Kaplan-Meier plots and the mean hospital stay was statistically compared using the Mantel-Cox log rank test. Data were analyzed using SPSS version 11 (SPSS Inc.) and GraphPad Prism version 6.0 (GraphPad Software). Comparison of the incidence of adverse events before and after intervention was statistically analyzed using McNemar's test for paired non-parametric variables. P-values of $<0.05$ was considered statistically significant.

\section{Results}

Patient demographics. A total of 484 (337 men and 147 women) patients who were hospitalized for the purpose of cancer chemotherapy were enrolled in the present study. Patient demographics are shown in Table I. The median age of patients was 66.0 years (5-95th, 56.0-76.0 years), and the mean duration of hospital stay was 12.3 days 
Table I. Patient demographics.

Characteristic

Number

Number of patients (male/female), $\mathrm{n}$

$484(337 / 147)$

Mean age (5-95th percentiles), years

$66.0(56.0-76.0)$

Mean length of hospital stay (5-95th percentiles), days

$12.3(4.0-24.0)$

Type of cancer, number of patients (\%)

Non-small cell lung cancer

$355(73.3)$

Small cell lung cancer

$110(22.7)$

Malignant meothelioma

$10 \quad(2.1)$

Thymoma/thymic carcinoma

Chemotherapy regimens, number of patients $(\%)$

Carboplatin (AUC 5) + pemetrexed $\left(500 \mathrm{mg} / \mathrm{m}^{2}\right) \pm$ bevacizumab $(15 \mathrm{mg} / \mathrm{kg})$

$88(18.2)$

Carboplatin (AUC 5) + paclitaxel $\left(200 \mathrm{mg} / \mathrm{m}^{2}\right) \pm$ bevacizumab $(15 \mathrm{mg} / \mathrm{kg})$

$85(17.5)$

Docetaxel $\left(60 \mathrm{mg} / \mathrm{m}^{2}\right)$

67 (13.8)

Amurubicin $\left(40 \mathrm{mg} / \mathrm{m}^{2}\right)$

$41(8.5)$

Carboplatin (AUC 5) + etoposide $\left(100 \mathrm{mg} / \mathrm{m}^{2}\right)$

$33 \quad(6.8)$

Pemetrexed $\left(500 \mathrm{mg} / \mathrm{m}^{2}\right)+$ bevacizumab $(15 \mathrm{mg} / \mathrm{kg})$

$30 \quad(6.2)$

Tyrosine kinase inhibitors against epidermal growth factor receptor

$23(4.8)$

Nogitecan $\left(1.0 \mathrm{mg} / \mathrm{m}^{2}\right)$

$15 \quad(3.1)$

Irinotecan $\left(100 \mathrm{mg} / \mathrm{m}^{2}\right)$

14 (2.9)

Cisplatin $\left(80 \mathrm{mg} / \mathrm{m}^{2}\right)+$ vinorelbine $\left(25 \mathrm{mg} / \mathrm{m}^{2}\right)$

$12(2.8)$

Vinorelbine $\left(25 \mathrm{mg} / \mathrm{m}^{2}\right)$

$9 \quad(1.8)$

Carboplatin (AUC 5) + tegafur/gimeracil/oteracil

$7 \quad(1.4)$

Cisplatin $\left(80 \mathrm{mg} / \mathrm{m}^{2}\right)+$ pemetrexed $\left(500 \mathrm{mg} / \mathrm{m}^{2}\right) \pm$ bevacizumab $(15 \mathrm{mg} / \mathrm{kg})$

$7 \quad(1.4)$

Gemcitabine $\left(1,000 \mathrm{mg} / \mathrm{m}^{2}\right)$

$7 \quad(1.4)$

Cisplatin $\left(80 \mathrm{mg} / \mathrm{m}^{2}\right)+$ docetaxel $\left(60 \mathrm{mg} / \mathrm{m}^{2}\right)$

$4(0.8)$

Carboplatin (AUC 5) + gemcitabine $\left(1,000 \mathrm{mg} / \mathrm{m}^{2}\right)$

$4(0.8)$

Tegafur/gimeracil/oteracil

$4 \quad(0.8)$

Other

$34(7.0)$

AUC, area under the curve.

(5-95th, 4.0-24.0 days). The most common cancer type was non-small cell lung cancer $(n=355,73.3 \%)$, followed by small cell lung cancer $(n=110,22.7 \%)$, malignant mesothelioma $(n=10,2.1 \%)$ and thymoma/thymic carcinoma $(n=9,1.9 \%)$. The most common cancer chemotherapy regimen was carboplatin $\left(\right.$ AUC 5) + pemetrexed $\left(500 \mathrm{mg} / \mathrm{m}^{2}\right) \pm$ bevacizumab $\left(15 \mathrm{mg} / \mathrm{m}^{2}\right)$ $(\mathrm{n}=88,18.2 \%)$, followed by carboplatin (AUC 5) + paclitaxel $\left(200 \mathrm{mg} / \mathrm{m}^{2}\right) \pm$ bevacizumab $\left(15 \mathrm{mg} / \mathrm{m}^{2}\right)(\mathrm{n}=85,17.5 \%)$, docetaxel $\left(60 \mathrm{mg} / \mathrm{m}^{2}\right)(\mathrm{n}=67,13.8 \%)$, amurubicin $\left(40 \mathrm{mg} / \mathrm{m}^{2}\right)$ $(\mathrm{n}=41,8.5 \%)$, carboplatin (AUC 5) + etoposide $\left(100 \mathrm{mg} / \mathrm{m}^{2}\right)$ $(\mathrm{n}=33,6.8 \%)$, and pemetrexed $\left(500 \mathrm{mg} / \mathrm{m}^{2}\right)+$ bevacizumab $\left(15 \mathrm{mg} / \mathrm{m}^{2}\right)(\mathrm{n}=30,6.2 \%)$.

Interventions implemented by the clinical pharmacist before administration of chemotherapy. Before administration of chemotherapy, the clinical pharmacist implemented a total of 152 interventions in 101 of the 484 (20.8\%) patients. The types of interventions implemented by the clinical pharmacist before administration of chemotherapy are shown in Table II. The most frequent type of intervention was drug addition $(n=82,53.9 \%)$, among which 76 interventions $(50.0 \%)$ were related to addition of supportive care, followed by dose adjustment $(n=27,17.8 \%)$, selection $(n=15$, $9.9 \%)$, discontinuation $(\mathrm{n}=12,7.9 \%)$, examination addition $(n=6,3.9 \%)$ and other $(n=10,6.6 \%)$.

Incidence of adverse events. As shown in Table III, there were a total of 365 adverse events, among which 29 (7.9\%), 227 (62.2\%), $89(24.4 \%)$, and $20(5.5 \%)$ were grade 1, 2, 3 and 4, respectively. A total of $203(41.9 \%)$ patients had adverse events, including $12(2.5 \%)$ with grade $1,106(21.9 \%)$ with grade $2,69(14.3 \%)$ with grade 3, 16 (3.3\%) with grade 4, and 191 (39.4\%) with grade $\geq 2$ events.

In contrast, the most common adverse event was nausea/vomiting $(23.4 \%)$, followed by neutropenia $(17.8 \%)$, constipation $(7.4 \%)$, anorexia $(6.2 \%)$, hepatic dysfunction/renal dysfunction (4.7\%), anemia (4.7\%), hyperglycemia $(3.8 \%)$, peripheral neuropathy $(3.8 \%)$, oral mucositis/esophagitis $(3.3 \%)$, myalgia/arthralgia/other pain (3.0\%), hiccups $(3.0 \%)$, diarrhea $(2.4 \%)$, dysgeusia $(2.1 \%)$, malaise $(1.8 \%)$ and thrombocytopenia (1.8\%) (Fig. 1A). The most common cause of adverse events was anticancer drugs $(71.7 \%)$, followed by other drugs $(23.5 \%)$, radiotherapy $(3.3 \%)$ and disease (1.5\%) (Fig. 1B). 
Table II. Drug-specific interventions by clinical pharmacist before administration of chemotherapy.

Category of intervention (n)

Contents of intervention (n)

Drug addition (82)

Drug dose adjustment (27)

Drug selection (15)

Drug discontinuation (12)

Examination addition (6)

Other (10)
Antiemetic drugs (26), vitamin B12 or folic acid on pemetrexed chemotherapy (18), zoledronic acid or precipitated calcium carbonate/cholecalciferol/magnesium carbonate for high blood calcium due to cancer (14), hydration on cisplatin chemotherapy or renal dysfunction (12), other drugs (12)

Anticancer drugs (22), other drugs (5)

Antiemetic drugs (7), anticancer drugs (8)

Antiemetic drugs (7), anticancer drug (2), other drugs (3)

Urinary protein examination on bevacizumab chemotherapy (3), other (3)

Drug-drug interactions (5), change of solution used to dissolve anticancer drugs (2), other (3)
Table III. Incidence of adverse events according to severity.

\begin{tabular}{lrr}
\hline Grade & Number & Patients, n (\%) \\
\hline Grade 1 & 29 & $12(2.5)$ \\
Grade 2 & 227 & $106(21.9)$ \\
Grade 3 & 89 & $69(14.3)$ \\
Grade 4 & 20 & $16(3.3)$ \\
All grades & 365 & $203(41.9)$ \\
Grade $\geq 2$ & 336 & $191(39.4)$ \\
\hline
\end{tabular}

As shown in Fig. 1C, the mean duration of hospitalization was significantly longer among patients with grade $\geq 2$ events than those with grade 0 and 1 events [7.8 days, range 2.5-18.7 days vs. 18.1 days, range 4.0-48.6 days; hazards ratio (HR) $0.457,95 \%$ confidence interval (CI) 0.377-0.555; $\mathrm{P}<0.001]$.

Recommendations by the clinical pharmacist for treatment of adverse events. There were 224 medical interventions and 73 follow ups for grade $\geq 2$ non-hematological or grade $\geq 3$ hematological adverse events. Seventy-seven of $170(45.3 \%)$ medical interventions for grade $\geq 2$ non-hematological adverse events were implemented based on the pharmacist's recommendations (Fig. 2A). In contrast, 4 of 54 (7.5\%) medical interventions for grade $\geq 3$ hematological adverse events were based on the pharmacist's recommendations (Fig. 2B).

The rates of pharmacist's recommendations in medical intervention for each of non-hematological adverse events of grade $\geq 2$ or hematological adverse events of grade $\geq 3$ observed was as follows: Peripheral neuropathy (92.3\%), followed by malaise $(75.0 \%)$, nausea/vomiting $(59.1 \%)$, oral mucositis/esophagitis $(54.5 \%)$, diarrhea $(50.0 \%)$, myalgia/arthralgia/other pain $(28.6 \%)$, constipation $(26.1 \%)$, hiccups $(20.0 \%)$, anorexia $(14.3 \%)$, hepatic/renal dysfunction $(11.1 \%)$, neutropenia $(8.2 \%)$ and hyperglycemia $(7.1 \%)$ (Fig. 2C).

Effect of medical interventions for adverse events. As shown in Fig. 3A, the incidence of grade $\geq 2$ non-hematological or grade $\geq 3$ hematological adverse events was significantly reduced after intervention (39.4 vs. $17.8 \%, \mathrm{P}<0.01)$. Moreover, as shown in Fig. 3B, the incidence rates of following non-hematological adverse events of grade $\geq 2$ or hematological adverse events of grade $\geq 3$ observed were significantly reduced after implementation of medical interventions listed in Table IV: Nausea/vomiting (16.3 vs. $4.5 \%, \mathrm{P}<0.01$ ), constipation (5.4 vs. $0.8 \%, \mathrm{P}<0.01$ ), anorexia (4.3 vs. $1.2 \%$, $\mathrm{P}<0.01$ ), hyperglycemia (3.3 vs. $1.7 \%, \mathrm{P}<0.01$ ), oral mucositis/esophagitis (3.1 vs. $1.7 \%, \mathrm{P}<0.05)$, hepatic or renal dysfunction ( 2.5 vs. $0.8 \%, \mathrm{P}<0.01)$, myalgia/arthralgia/other pain (2.1 vs. $0.2 \%, \mathrm{P}<0.01)$, hiccups (2.1 vs. $0.4 \%, \mathrm{P}<0.01)$, diarrhea (1.2 vs. $0 \%, \mathrm{P}<0.05)$, malaise $(1.2$ vs. $0 \%, \mathrm{P}<0.05)$ and neutropenia (10.5 vs. $0.6 \%, \mathrm{P}<0.01)$.

\section{Discussion}

Here, we report a clinical pharmacist's interventions for the prevention and treatment of adverse events in hospitalized patients with thoracic cancer receiving cancer chemotherapy. Before administration of cancer chemotherapy, the clinical pharmacist performed a total of 152 interventions for $23.6 \%$ of eligible patients, including drug addition (53.9\%), dose adjustment (17.8\%), selection (9.9\%), discontinuation $(7.9 \%)$, examination addition (3.9\%), and drug-drug interactions $(3.3 \%)$. Therefore, half of the interventions involved addition of a drug for the prevention of adverse events.

Several studies have reported the efficacy of clinical pharmacists using physician-approved protocols in pharmaceutical care (18-20). In the present study, interventions were implemented by a single clinical pharmacist before administration of cancer chemotherapy based on a treatment protocol agreed upon by physicians and clinical pharmacists in advance. This protocol set the criteria for starting treatment, dose reduction, drug withdrawal based mainly on evidence from original reports, and prophylactic supportive care. As a result, all patients received the appropriate dosage of anticancer drug and prophylactic supportive therapy based on the treatment protocol agreed upon by physicians and clinical pharmacists in advance.

Despite implementation of the appropriate dosage of anticancer drug and prophylactic supportive therapy with above mention, 39.4\% (191/484) of patients suffered grade $\geq 2$ non-hematological or grade $\geq 3$ hematological adverse events, and the most common adverse events was nausea/vomiting, followed by neutropenia, constipation, anorexia, hepatic dysfunction/renal 

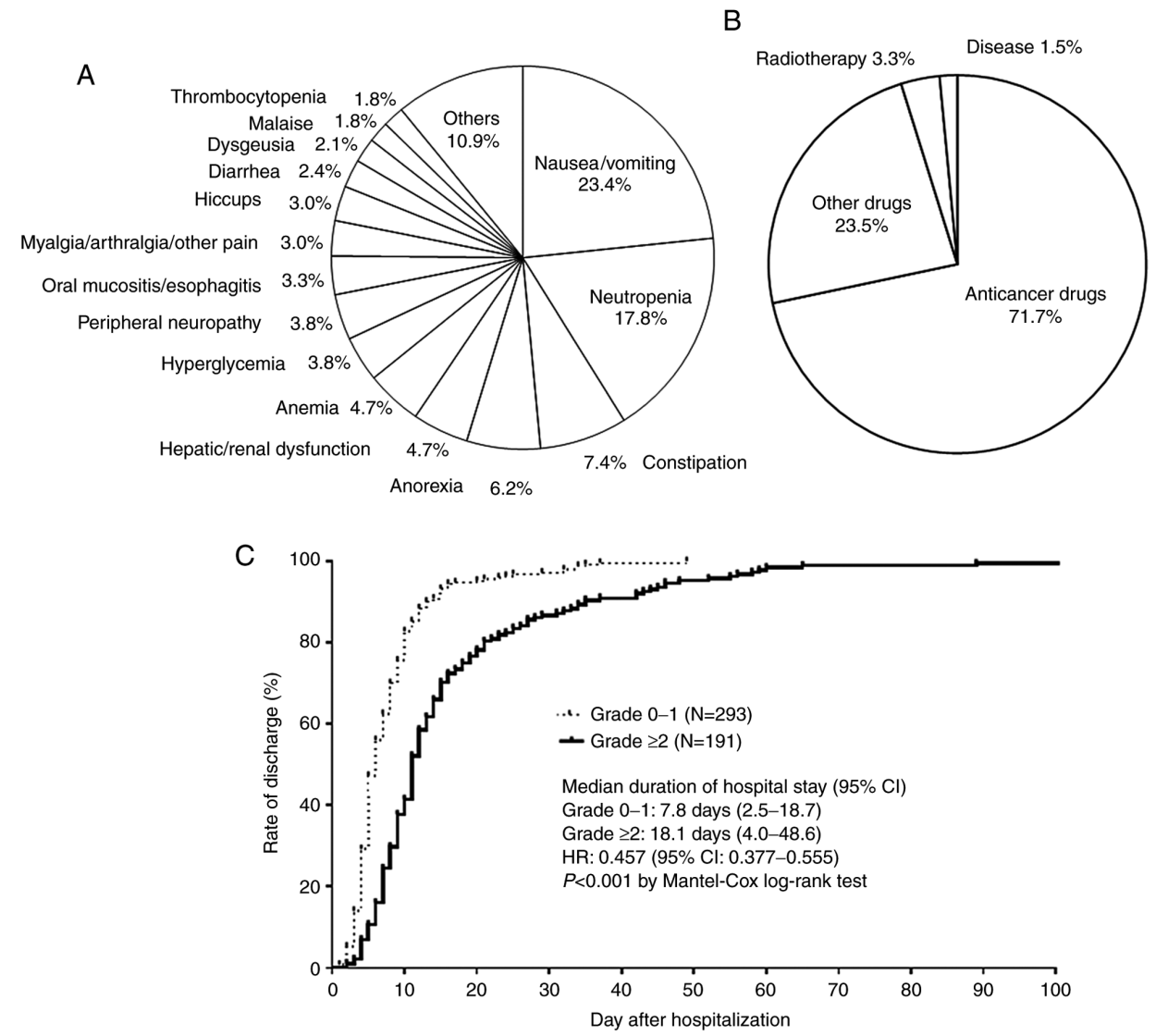

Figure 1. Incidence and influence of adverse events. (A) Types of adverse events, (B) causes of adverse events and (C) duration of hospital stay of patients with either grade $0-1$ or grade $\geq 2$ adverse events. Data were statistically compared using Mantel-Cox log rank test.

A

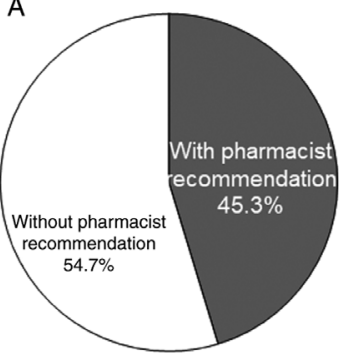

C

With pharmacist recommendation $(\%)$

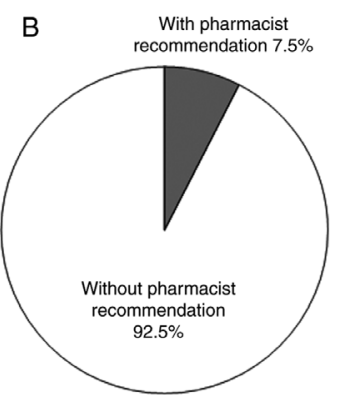

$\square$ Without pharmacist recommendation (\%)

\begin{tabular}{|c|c|c|}
\hline \multirow{2}{*}{$\begin{array}{r}\text { Peripheral neuropathy } \\
\text { Malaise }\end{array}$} & \multicolumn{2}{|c|}{92.3} \\
\hline & 75.0 & 25.0 \\
\hline Nausea/vomiting & 59.1 & 40.9 \\
\hline Oral mucositis/esophagitis & 54.5 & 45.5 \\
\hline Diarrhea & 50.0 & 50.0 \\
\hline Myalgia/arthralgia/other pain & 28.6 & 71.4 \\
\hline Constipation & 26.1 & 73.9 \\
\hline Hiccups & \multicolumn{2}{|r|}{80.0} \\
\hline Anorexia & \multicolumn{2}{|c|}{85.7} \\
\hline Hepatic/renal dysfunction & \multicolumn{2}{|c|}{88.9} \\
\hline Hyperglycemia & \multicolumn{2}{|c|}{92.9} \\
\hline Neutropenia & \multicolumn{2}{|c|}{91.8} \\
\hline Thrombocytopenia & \multicolumn{2}{|c|}{100} \\
\hline Anemia & \multicolumn{2}{|c|}{100} \\
\hline
\end{tabular}

Figure 2. Intervention for adverse events. The rates of pharmacist's recommendations in medical intervention for (A) grade $\geq 2$ non-hematological adverse events, (B) grade $\geq 3$ hematological adverse events and (C) each non-hematological adverse event of grade $\geq 2$ or hematological adverse event of grade $\geq 3$. 
Table IV. Contents of medical interventions for grade $\geq 2$ adverse events with highest incidence.

Adverse event (n)

Nausea/vomiting (66)

Constipation (33)

Anorexia (21)

Hepatic or renal dysfunction (13)

Hyperglycemia (14)

Peripheral neuropathy (13)

Oral mucositis/esophagitis (8)

Myalgia/arthralgia/other pain (9)

Hiccups (11)

Diarrhea (6)

Malaise (3)

Neutropenia (55)

Anemia (1)

Thrombocytopenia (1)
Intervention (n)

Olanzapine (34), prochlorperazine (18), metoclopramide (3), granisetron (3), other (8) Magnesium oxide (11), sennoside (8), bisacodyl (8), picosulfate (4), glycerine(2)

Follow up (16), prochlorperazine (3), dexametasone (2)

Hepatic dysfunction: Discontinuation of the suspected drug (3), glycyrrhizinate (3), renal dysfunction: Hydration (7)

Insulin (14)

Pregabalin (11), duloxetine (2)

Steroids (4), polaprezinc (3), acetaminophen (1)

Loxoprofen (4), shakuyaku-kanzo-to (1), follow up (4)

Clonazepam (5), chlorpromazine (3), prochlorperazine (2), shakuyaku-kanzo-to (1)

Loperamide (2), trimebutine (2), hange-shashin-to (2)

Steroids (3)

G-CSF (55)

Blood transfusion (1)

Blood transfusion (1)

G-CSF, granulocyte-colony stimulating factor.

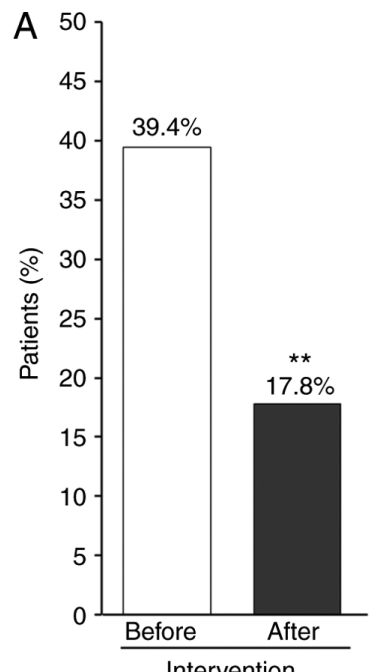

B

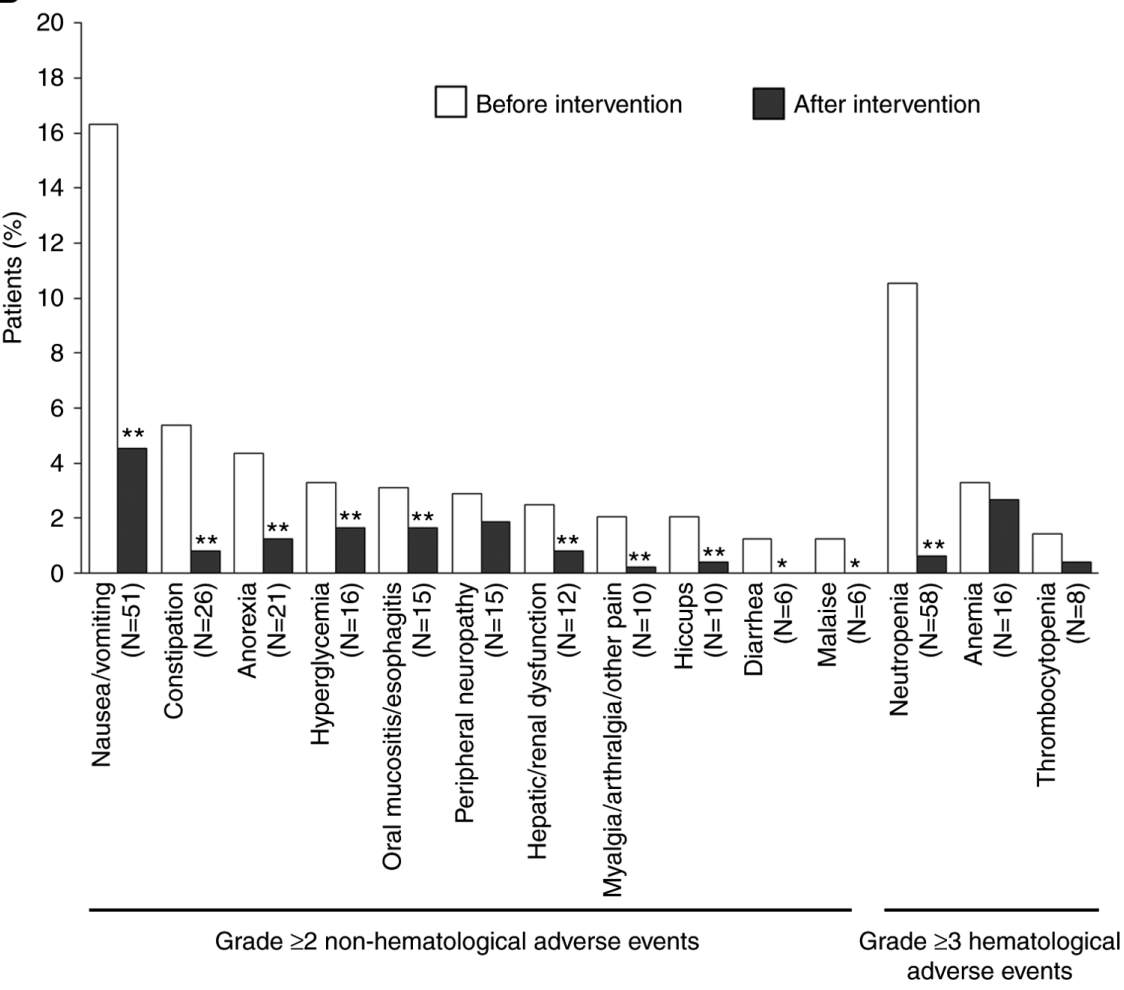

Figure 3. Effects of medical interventions. Effect of medical interventions on the (A) incidence of grade $\geq 2$ non-hematological or grade $\geq 3$ hematological adverse events and (B) incidence of each adverse event of grade $\geq 2$ non-hematological or grade $\geq 3$ hematological adverse events. McNemar's test was used to analyze data. ${ }^{*} \mathrm{P}<0.05$ and ${ }^{* *} \mathrm{P}<0.01$ vs. before intervention.

dysfunction, anemia, hyperglycemia, peripheral neuropathy, oral mucositis/esophagitis, myalgia/arthralgia/other pain, hiccups, diarrhea, dysgeusia, malaise and thrombocytopenia. Previous studies have reported a high incidence of adverse events in patients who received cancer chemotherapy $(21,22)$.
Borghaei et al reported that $41 \%(1,624 / 3,967)$ of elderly patients with advanced non-small cell lung cancer on second-line therapy experienced one or more severe adverse events, of which hypertension, anemia, and pneumonia were most common (21). In another report, the incidence of chemotherapy-related adverse 
events in 1,682 patients with metastatic breast cancer was $54 \%$, and the most common adverse events categories were hematological, musculoskeletal/pain related, gastrointestinal and infection/pyrexia (22).

Our previous findings indicated that implementation of medical intervention significantly lowered the incidence of a variety of adverse events in inpatients with head and neck cancer, including microbial infection, oral mucositis, odynophagia, neutropenia, insomnia, and constipation (23). Consistent with this, the present study showed that implementation of medical interventions significantly reduced the incidence of a variety of adverse events. Among these, $45.3 \%$ of medical interventions for non-hematological adverse events were implemented based on the pharmacist's recommendations. Although only $7.5 \%$ of interventions for hematological adverse events were based on the clinical pharmacist's recommendations, the clinical pharmacist monitored absolute neutrophil counts and platelet and hemoglobin levels to assure blood parameters were within acceptable limits for the next cycle of chemotherapy. The pharmacist's recommendations for medical interventions primarily contributed to improving peripheral neuropathy, followed by malaise, diarrhea, oral mucositis/esophagitis, nausea/vomiting, constipation, hiccups and myalgia/arthralgia/other pain. Additionally, the medical interventions significant improved these adverse events, except for peripheral neuropathy. At present, there are no sufficient treatment options available for chemotherapy-induced peripheral neuropathy because the exact pathophysiology is unclear (24).

Several lines of evidence indicate that the incidence of moderate-to-severe adverse events is associated with prolonged hospital stay (25-27). In the present study, the mean time to discharge was markedly different between patients with grade 0 and 1 events and those with grade $\geq 2$ events. Therefore, prevention or relief of adverse events may lead to reduced hospitalization periods.

There are several limitations in the present study. First, this was a non-randomized single-centre retrospective study. Second, the sample size was small, limiting our ability to detect statistically significant differences in the data. Third, the patient population was limited to inpatients with thoracic cancer receiving cancer chemotherapy. Therefore, a large-scale, multi-institutional prospective study is required to confirm our present findings.

In conclusion, we found that a clinical pharmacist performed interventions in $23.6 \%$ of eligible patients before administration of cancer chemotherapy. Although all patients received the appropriate dosage of anticancer drug and prophylactic supportive therapy based on the agreed treatment protocol, $39.4 \%$ of patients suffered grade $\geq 2$ non-hematological or grade $\geq 3$ hematological adverse events. Implementation of medical interventions significantly reduced the incidence of adverse events, among which 45.3 and $7.5 \%$ of medical interventions for non-hematological and hematological adverse events, respectively, were implemented based on the pharmacist's recommendations. One pharmacist in the respiratory medicine ward therefore made a marked contribution to preventing and relieving adverse events in hospitalized patients with thoracic cancer receiving cancer chemotherapy.

\section{Acknowledgements}

The authors would like to thank Ms. Maya Tatsumi-Yamada (Gifu University Hospital, Gifu, Japan) for the assistance in the study.

\section{Funding}

No funding was received.

\section{Availability of data and materials}

The datasets used and/or analyzed during the present study are available from the corresponding author on reasonable request.

\section{Authors' contributions}

$\mathrm{HI}, \mathrm{CH}, \mathrm{AS}$ and $\mathrm{YO}$ conceived and designed the study. $\mathrm{HI}, \mathrm{CH}, \mathrm{NF}, \mathrm{JE}, \mathrm{FI}, \mathrm{KY}$ and DK were involved with the acquisition of data. HI, AS and YO analyzed and interpreted the data. HI, CH, JE and YO assessed the authenticity of all the raw data. HI, CH, NF, JE, FI, KY, DK, YO and AS drafted the manuscript and revised it critically for important intellectual content. All authors read and approved the final manuscript.

\section{Ethics approval and consent to participate}

The present study was conducted according to the guidelines for human studies of the ethics committee of Gifu University Graduate School of Medicine and the Government of Japan, and was approved by the university's institutional review board (approval no. 28-348). In view of the retrospective nature of the study, informed consent from the subjects was not mandated.

\section{Patient consent for publication}

Not applicable.

\section{Competing interests}

The authors declare that they have no competing interests.

\section{References}

1. Vincent $\mathrm{C}$, Neale $\mathrm{G}$ and Woloshynowych M: Adverse events in British hospitals: Preliminary retrospective record review. BMJ 322: 517-519, 2001.

2. Baker GR, Norton PG, Flintoft V, Blais R, Brown A, Cox J, Etchells E, Ghali WA, Hébert P, Majumdar SR, et al: The Canadian adverse events study: The incidence of adverse events among hospital patients in Canada. CMAJ 170: 1678-1686, 2004.

3. Hurvitz S, Guerin A, Brammer M, Guardino E, Zhou ZY, Latremouille Viau D, Wu EQ and Lalla D: Investigation of adverse-event-related costs for patients with metastatic breast cancer in a real world setting. Oncologist 19: 901-908, 2014.

4. Wong W, Yim YM, Kim A, Cloutier M, Gauthier-Loiselle M, Gagnon-Sanschagrin P and Guerin A: Assessment of costs associated with adverse events in patients with cancer. PLoS One 13: e0196007, 2018.

5. Rashid N, Koh HA, Baca HC, Li Z, Malecha S, Abidoye O and Masaquel A: Clinical impact of chemotherapy-related adverse events in patients with metastatic breast cancer in an integrated health care system. J Manag Care Spec Pharm 21: 863-871, 2015. 
6. Caggiano V, Weiss RV, Rickert TS and Linde-Zwirble WT: Incidence, cost, and mortality of neutropenia hospitalization associated with chemotherapy. Cancer 103: 1916-1924, 2005.

7. Shune SE, Karnell LH, Karnell MP, Van Daele DJ and Funk GF Association between severity of dysphagia and survival in patients with head and neck cancer. Head Neck 34: 776-784, 2012.

8. Mathijssen RH, Sparreboom A and Verweij J: Determining the optimal dose in the development of anticancer agents. Nat Rev Clin Oncol 11: 272-281, 2014.

9. Ranchon F, Salles G, Späth HM, Schwiertz V, Vantard N, Parat S, Broussais F, You B, Tartas S, Souquet PJ, et al: Chemotherapeutic errors in hospitalised cancer patients: Attributable damage and extra costs. BMC Cancer 11: 478, 2011.

10. Phillips J, Beam S, Brinker A, Holquist C, Honig P, Lee LY and Pamer C: Retrospective analysis of mortalities associated with medication errors. Am J Health Syst Pharm 58: 1835-1841, 2001

11. Chewning B and Wiederholt JB: Concordance in cancer medication management. Patient Educ Couns 50: 75-78, 2003.

12. Strasser F, Sweeney C, Willey J, Benisch-Tolley S, Palmer JL and Bruera E: Impact of a half-day multidisciplinary symptom control and palliative care outpatient clinic in a comprehensive cancer center on recommendations, symptom intensity, and patient satisfaction: A retrospective descriptive study. J Pain Symptom Manage 27: 481-491, 2004.

13. Iihara H, Ishihara M, Matsuura K, Kurahashi S, Takahashi T, Kawaguchi Y, Yoshida K and Itoh Y: Pharmacists contribute to the improved efficiency of medical practices in the outpatient cancer chemotherapy clinic. J Eval Clin Pract 18: 753-760, 2012.

14. Shah S, Dowell J and Greene S: Evaluation of clinical pharmacy services in a hematology/oncology outpatient setting. Ann Pharmacother 40: 1527-1533, 2006.

15. Han JM, Ah YM, Suh SY, Jung SH, Hahn HJ, Im SA and Lee JY: Clinical and economic impact of pharmacists' intervention in a large volume chemotherapy preparation unit. Int J Clin Pharm 38 1124-1132, 2016.

16. Shah S, Dowell J and Greene S: Evaluation of clinical pharmacy services in a hematology/oncology outpatient setting. Ann Pharmacother 40: 1527-1533, 2016.

17. Suzuki H, Suzuki S, Kamata H, Sugama Y, Demachi K, Ikegawa K, Igarashi $\mathrm{T}$ and Yamaguchi M: Impact of pharmacy collaborating services in an outpatient clinic on improving adverse drug reactions in outpatient cancer chemotherapy. J Oncol Pharm Pract 25: 1558-1563, 2018.
18. Martin JK Jr and Norwood MB: Pharmacist management of antiemetic therapy under protocol in an oncology clinic. Am J Hosp Pharm 45: 1322-1328, 1988.

19. Horne AL and Dapolite LA: Protocol for pharmacist management of antineoplastic drug-induced adverse effects in outpatients. Am J Health Syst Pharm 54: 680-683, 1997.

20. Gums TH, Uribe L, Vander Weg MW, James P, Coffey C and Carter BL: Pharmacist intervention for blood pressure control: Medication intensification and adherence. J Am Soc Hypertens 9: 569-578, 2015.

21. Borghaei H, Yim YM, Guerin A, Pivneva I, Shi S, Gandhi M and Ionescu-Ittu R: Severe adverse events impact overall survival and costs in elderly patients with advanced non-small cell lung cancer on second-line therapy. Lung Cancer 119: 112-119, 2018.

22. Rashid N, Koh HA, Baca HC, Lin KJ, Malecha SE and Masaquel A: Economic burden related to chemotherapy-related adverse events in patients with metastatic breast cancer in an integrated health care system. Breast Cancer 8: 173-181, 2016.

23. Suzuki A, Kobayashi R, Okayasu S, Kuze B, Aoki M, Mizuta K and Itoh Y: Pharmacotherapy for adverse events reduces the length of hospital stay in patients admitted to otolaryngology ward: A single arm intervention study. PLoS One 9: e115879, 2014.

24. Hou S, Huh B, Kim HK, Kim KH and Abdi S: Treatment of chemotherapy-induced peripheral neuropathy: Systematic review and recommendations. Pain Physician 21: 571-592, 2018.

25. Classen DC, Pestotnik SL, Evans RS, Lloyd JF and Burke JP: Adverse drug events in hospitalized patients. Excess length of stay, extra costs, and attributable mortality. JAMA 277: 301-306, 1997.

26. Bates DW, Spell N, Cullen DJ, Burdick E, Laird N, Petersen LA, Small SD, Sweitzer BJ and Leape LL: The costs of adverse drug events in hospitalized patients. JAMA 277: 307-311, 1997.

27. Nishida S, Hayashi Y, Suzuki A, Kobayashi R, Inuzuka T and Itoh Y: Relationship between number of drugs and duration of hospital stay in older patients with neuromuscular diseases. Geriatr Gerontol Int 18: 1018-1024, 2018. 